\title{
Moral Leadership and Effectiveness of Enterprise Innovation: Meta-Analysis Based on Multifocus Method
}

\author{
Bo Lin ${ }^{1, a}$, Siyuan Chen ${ }^{2, b}$ and Tielin Zhao ${ }^{3, c, *}$ \\ ${ }^{1,2,3}$ School of Economics and Management Dalian University No.10, Xuefu Street, Jinzhou New \\ District, Dalian 116622, P. R. China \\ alinbo@dlu.edu.cn, ${ }^{b}$ cicichen1002@163.com, czhaotielin@dlu.edu.cn \\ ${ }^{*}$ Corresponding author
}

Keywords: Moral leadership, Innovation behavior, Meta-analysis

\begin{abstract}
The research is based on 54 empirical studies (56 effect values, total sample 21900) that were conducted to study the relationship between moral leadership and innovation effectiveness of multi-focus enterprises in paternalistic leadership by using meta-analysis technology. Moral leadership in paternalistic leadership has a significant impact on the effectiveness of enterprise innovation. Based on the multi-focus perspective, the implementation of enterprise innovation effectiveness includes many aspects, such as employee innovation ability and employee innovation behavior. That is to say, the performance of enterprise innovation effectiveness is based on the development of employee innovation ability and employee innovation behavior. Therefore, the effectiveness of enterprise innovation under the paternalistic leadership can be divided into: innovation ability orientation and innovation behavior orientation. In the context of moral leadership, it is not yet clear which subject employees are more inclined to innovate. Research shows that moral leadership has significant positive effects on innovation behavior, capability oriented innovation and behavior oriented innovation. The cross-cultural situation (China, non -China) and the innovation subject (individual, team, organization) regulate the relationship between them.
\end{abstract}

\section{德行领导与企业创新有效性：基于多焦点法的Meta分析 \\ 林波 ${ }^{1, a}$, 陈思缘 ${ }^{2, b}$, 赵铁林 $^{3, \mathrm{c},{ }^{*}}$ \\ $1,2,3$ 大连大学经济管理学院, 大连, 辽宁, 中国 \\ alinbo@dlu.edu.cn, ${ }^{\mathrm{b}}$ cicichen1002@163.com, ${ }^{\mathrm{C}}$ zhaotielin@dlu.edu.cn \\ “通讯作者}

关键词：德行领导; 创新行为; Meta分析

中文摘要.本文以54篇实证研究 (56个效应值, 样本总量21900)为对象, 运用Meta分析技术研 究家长式领导中德行领导与多焦点企业创新有效性的关系。家长式领导中的德行领导对企业 创新有效性具有显著的影响。基于多焦点视角, 企业创新有效性的实施内容包括员工创新能 力、员工创新行为多个方面, 即企业创新有效性能以员工创新能力、员工创新行为的发展作 为基础, 因此，在家长式领导下的企业创新有效性可分为：创新能力导向、创新行为导向。 在德行领导情境下, 员工针对哪个主体实施创新行为的倾向更强, 尚无定论。研究表明, 德 行领导对企业创新行为、能力导向企业创新、行为导向企业创新具有显著的正效应。跨文化 情境(中国、非中国)、创新主体（个体、团队、组织）对两者关系起调节作用。 


\section{1. 引言}

近些年, 随着 “大众创业, 万众创新”思想潮流的发展, 创新领域研究越来越受到学者 关注, 但以往大多关注于科技水平的创新, 对管理创新领域的研究力度不足, 其中管理创新 在近十多年才逐渐受到重视 ${ }^{[1]}$ 。管理创新之所以受到重视, 主要源自其对组织发展的积极作 用。管理创新被认为是 21 世纪公司成功的关键, 学者们对德行领导的研究兴趣不断地提升 ${ }^{[2,3,4]}$, 许多学者认为德行领导, 对企业创新有重要影响, 德行领导更是成为最受学者关注的领导行 为之一, 通过对大量文献的梳理, 发现家长式领导中德行领导与多焦点企业创新有效性有显 著的影响效果, 本文旨在用Meta分析定量评价现有研究, 通过纠正样本和测量误差得到德行 领导与企业创新有效性更具普遍性的结论，并为相关研究和决策提供借鉴。

\section{2. 理论回顾与假设}

领导方式是感知企业创新行为的重要情境因素, 德行领导是家长式领导的重要组成部分, 对企业创新有重要影响。德行领导是指领导者通过在人际交往以及日常工作中展现的道德素 质和人格鬼米力, 特别是真诚友善、对下属一视同仁、关怀下属发展等的树德行为, 以赢得下 属的支持、效仿 ${ }^{[5]}$ 。根据社会交换理论 ${ }^{[6]}$ 和互惠规范 ${ }^{[7]}$, 当领导者采取德行领导时, 使下属感 受到关怀、公平的待遇，下属会主动与自己的领导形成稳定的社会交换关系，并且为了回报 领导的赏识, 员工会采取更加积极的工作行为 ${ }^{[8,9,10]}$, 大多数研究表明家长式领导中德行领导 对企业创新、创造力等相关概念具有显著的正向影响 ${ }^{[11,12,13,14]}$ 。比如Dedahanov, A.T (2016) 曾经研究了德行领导与员工创造力之间的关系, 并得出研究结论: 二者之间的影响关系显著, 也有极少部分的学者认为德行领导对企业创新有效性会产生负向的影响效果 ${ }^{[15]}$, 因此对于德 行领导与企业创新的探索还应该进一步加深。

德行领导在世界范围内已经广泛存在，许多学者已经从不同角度不同方面对德行领导的 有效性和适用性进行了探讨和验证。虽然对于德行领导的研究最早是从西方开始的, 但是作 为一种孕育于东方文化中的领导方式, 多项研究表明, 相比较西方国家, 德行领导在中国情 境下表现出更高的契合度, 具有更高的领导效能。中国台湾学者郑伯埙（2000）通过对已有 德行领导的研究进行总结, 证实了德行领导广泛存在于现代组织当中, 在不同的情境下德行 领导的领导效能是否会有所不同? 在本研究中, 将引入跨文化情境来探讨德行领导对企业创 新的影响是否会受到中国情境和非中国情境的影响。

个体是实践活动的承担者。创新主体是自始至终参与到创新的全过程，有自己的创意想 法并将其付诸实践的人。根据企业创新所实施的创新内容可以分为: 理论创新、技术创新、 制度创新等。不同的创新主体在创新过程中所具备的创新素质和创新能力往往是不同的。因 而, 不同的创新素质和创新能力对企业的总体创新也会起到不同程度的影响。根据创新实施 的主体不同，员工会根据面临不同的处境、场景选取不同的最佳焦点行为 ${ }^{[16,17]}$, 在统计相关 文献的基础上, 本研究将企业创新的导向根据多焦点法分为能力导向、行为导向企业创新。 创新主体分为个体、团队和组织三个层面。

基于以上分析, 本研究提出研究假设H1-H4.

H1：德行领导与企业总体创新显著正相关。

H1a: 德行领导与能力导向企业创新显著正相关。

$\mathrm{H} 1 b$ : 德行领导与行为导向企业创新显著正相关。

$\mathrm{H} 2$ : 德行领导对能力导向创新行为的影响高于对行为导向创新行为的影响。

H3: 跨文化情境在德行领导与多焦点创新行为的关系中起调节效应, 在非中国情境下, 德行领导对企业创新、能力导向企业创新、行为导向企业创新的影响更弱。

H4: 创新主体在德行领导与企业创新的关系中起调节作用, 德行领导对创新主体为团队 的企业总体创新、能力导向企业创新的影响更强。 


\section{3. 研究方法}

\section{1 文献检索}

采用多种方式收集德行领导、家长式领导、企业创新的中英文实证研究文献。首先, 检 索中国知网、Google Scholar等常用中英文数据库, 关键词有: “德行领导” “家长式领导” “中国式领导” “创新” “企业创新行为” “创造力” “创新行为” “moral leadership” “paternalistic leadership” “transformational leadership” "leadership style” "creativity” "innovative behavior” 等; 其次, 人工检索相关期刊, 如管理学报、南开管理评论、Management Decision、Journal of Management \& Organization。最后，查阅相关元分析文章的参考文献进一步补充文献。

\section{2 文献管选}

本文按照以下标准确定最终纳入元分析的文献：（1）研究是实证研究；（2）研究的前 因变量必须是德行领导或包含德行领导, 结果变量必须反映企业创新：（3）文章必须汇报德 行领导或包含德行领导与企业创新的相关系数 $r$ 或者可以转化为相关系数 $r$ 的统计量; (4) 文 献报告中两个变量所构成的效应值个数不少于1个;（5）研究文献必须是独立样本。通过以 上篮选标准，最后共有54篇文章纳入本研究。

\section{3 文献编码及分析技术}

研究共包括54篇文献，经过编码后得到56个效应值( 样本总量为21900)。文献编码内容 包括样本特征和效应值两个方面, 其中样本特征包括作者、发表年份、发表期刊、样本数量、 跨文化情境( 中国、非中国) 、创新主体( 个体、团队、组织)等。效应值包括：德行领导或包 含德行领导与企业创新的相关系数及其显著水平, 在德行领导与总体企业创新相关系数的编 码过程中, 利用文献提供的相关系数进行编码。为保证编码的准确性, 由两位作者先后两次 对本文进行编码, 其一致性达98\%以上。本研究采用 CMA2.0( comprehensive meta -analysis) 软件进行数据处理。

\section{4. 研究结果}

\section{1 异质性及发表偏差检验}

异质性说明研究结论的变异来源于不同研究设计多于抽样误差, 通过组内 $Q$ 统计量检 验其统计显著性, 以 $I^{2}$ 指标表示实际显著性。结果如表1所示, 在企业创新的研究中, $Q$ 统计 量在 0.001 水平上显著, 说明各研究的效应量存在异质性, 应采用随机模型分析方法。 $I^{2}$ 的 值为 93.488 , 说明 $93.488 \%$ 的观察变异来源于真实差异, 随机误差只能解释观察变异的 $6.512 \%$ 。 $\mathrm{Tau}^{2}$ 的值为 0.039 , 说明研究间变异有 $3.9 \%$ 可用来计算权重。针对能力导向、行为导向企业创 新的分析同上。

表1 效应量异质性检验结果

\begin{tabular}{|l|c|c|c|c|c|c|c|c|}
\hline 领导方式 & 结果变量 & $K$ & $Q$ & $d f$ & $I^{2}$ & Tau $^{2}$ & 失安全系数 & 未出版文献 \\
\hline \multirow{3}{*}{ 德行领导导 } & 企业创新 & 54 & $875.246^{* * *}$ & 57 & 93.488 & 0.039 & 47.198 & 3577 \\
\cline { 2 - 9 } & 能力导向 & 41 & $601.139^{* * *}$ & 42 & 92.841 & 0.040 & 42.421 & 101 \\
\cline { 2 - 9 } & 行为导向 & 15 & $267.842^{* * *}$ & 14 & 94.773 & 0.042 & 20.987 & 1705 \\
\hline
\end{tabular}

注: $p^{*}<0.05 ; p^{* *}<0.01 ; p^{* * *}<0.001$, 下同。

通常结果显著的文章更易被发表, 结果不显著的文章难以发表, 所以, 已发表文章的效 应值可能无法代表真实值, 因此, 在元分析研究中应检验发表偏差。本文采用失安全系数作 为检验标准, 失安全系数指再纳入多少文献才能使现有研究结果不显著, 若失安全系数非常 
小, 可能存在发表偏差。本研究结果如表1所示, 即要为每个主题研究分别找到3577、101、 1705项未出版的文献, 才能使本研究结果不显著, 因此, 本研究存在发表偏差的可能性极小。

\section{2 主效应检验}

结果如表 2 所示, 德行领导与企业总体创新的相关为 0.319 ，在 0.001 水平上显著, 故 假设 1 得到支持; 德行领导与能力导向、行为导向创新行为的相关分别为 $0.344 、 0.345$, 均 在在 0.001 水平上显著, 假设 $1 \mathrm{a} 、 1 \mathrm{~b}$ 得到支持。其中, 从点估计结果看, 德行领导与能力 向创新行为的相关性较强 $(r=0.344, p<0.001)$, 与行为导向创新行为的相关性较弱 $(r=0$. $245, p<0.001)$ ，故假设 2 得到支持。

表2 家长式领导与企业创新行为的主效应结果

\begin{tabular}{|c|c|c|c|c|c|c|c|}
\hline 领导方式 & 结果变量 & $K$ & $N$ & 点估计 & 下限 & 上限 & $Z$ Z值 \\
\hline \multirow{3}{*}{ 德行领导 } & 企业创新 & 54 & 21900 & 0.319 & 0.270 & 0.365 & $12.162^{* * *}$ \\
\cline { 2 - 8 } & 能力导向 & 41 & 14334 & 0.344 & 0.288 & 0.397 & $11.292^{* * *}$ \\
\cline { 2 - 8 } & 行为导向 & 15 & 7566 & 0.245 & 0.141 & 0.343 & $4.559^{* * *}$ \\
\hline
\end{tabular}

\section{3 调节效应检验}

即便效应值中存在微小差异, 也可通过判断 $Q$ 统计量是否显著, 来判断研究中是否存在 调节效应 ${ }^{[18]}$ 。研究结果如表3所示, 表3中 $Q$ 统计量均显著, 则在德行领导与企业创新中存在 跨文化情境、创新主体的调节变量, 在中国文化情境下, 德行领导与企业总体创新、能力导 向企业创新的相关分别为 $0.323 、 0.350$, 显著高于其在非中国文化情境下的相关性 $(r=0.295$, $p<0.001) 、(r=0.316, p<0.001)$, 但在非中国情景下, 德行领导对行为导向企业创新作 用效果不显著 $(Z$ 值 $=1.577, \mathrm{p}>0.05)$ ，因此，假设 3 没有得到支持。创新主体在德行领导与 各结果变量的关系中起调节作用, 德行领导与团队企业总体创新 $(r$ 才 $=0.440>r$ 组 $=0.371$ $>r$ 个 $=0.269, p<0.001)$ 、团队能力导向企业创新 $(r$ 才 $=0.440>r$ 组 $=0.371>r$ 个 $=0.285$, $p<0.001))$ 的相关性最强, 由于样本量的限制, 创新主体对德行领导与行为导向企业创新的 调节效果无法评价。故假设4没有得到支持。

表3 德行领导的相关变量调节效应结果

\begin{tabular}{|c|c|c|c|c|c|c|c|c|}
\hline 结果变量 & 调节变量 & $K$ & $N$ & 点估计 & 下限 & 上限 & Z值 & $Q$ \\
\hline \multirow{7}{*}{ 企业创新 } & 跨文化情境 & & & & & & & \\
\hline & 中国 & 44 & 18607 & 0.323 & 0.270 & 0.375 & $11.199^{* * *}$ & $741.157^{* * *}$ \\
\hline & 非中国 & 10 & 3293 & 0.295 & 0.171 & 0.409 & $4.546^{* * *}$ & $128.216^{* * *}$ \\
\hline & 创新主体 & & & & & & & \\
\hline & 个体 & 36 & 14947 & 0.269 & 0.210 & 0.326 & $8.683^{* * *}$ & $518.620^{* * *}$ \\
\hline & 团队 & 10 & 3724 & 0.440 & 0.316 & 0.548 & $6.397^{* * *}$ & $174.996^{* * *}$ \\
\hline & 组织 & 9 & 3229 & 0.371 & 0.305 & 0.434 & $10.158^{* * *}$ & $40.749^{* * *}$ \\
\hline \multirow{7}{*}{ 能力导向 } & 跨文化情境 & & & & & & & \\
\hline & 中国 & 33 & 11591 & 0.350 & 0.287 & 0.409 & $10.287^{* * *}$ & $479.249^{* * *}$ \\
\hline & 非中国 & 8 & 2743 & 0.316 & 0.179 & 0.442 & $4.372^{* * *}$ & $104.416^{* * *}$ \\
\hline & 创新主体 & & & & & & & \\
\hline & 个体 & 23 & 7381 & 0.285 & 0.211 & 0.355 & $7.298^{* * *}$ & $250.213^{* * *}$ \\
\hline & 团队 & 10 & 3724 & 0.440 & 0.316 & 0.548 & $6.397^{* * *}$ & $174.996^{* * *}$ \\
\hline & 组织 & 9 & 3229 & 0.371 & 0.305 & 0.434 & $10.158^{* * *}$ & $40.749^{* * *}$ \\
\hline \multirow{5}{*}{ 行为导向 } & 跨文化情境 & & & & & & & \\
\hline & 中国 & 13 & 7016 & 0.251 & 0.139 & 0.357 & $4.302^{* * *}$ & $246.298^{* * *}$ \\
\hline & 非中国 & 2 & 550 & 0.202 & -0.05 & 0.430 & 1.577 & $7.588^{* * *}$ \\
\hline & 创新主体 & & & & & & & \\
\hline & 个体 & 15 & 7566 & 0.245 & 0.141 & 0.343 & $4.559^{* * *}$ & $267.842^{* * *}$ \\
\hline
\end{tabular}




\section{5. 研究结论与展望}

本文运用Meta分析技术研究德行领导对企业创新有效性的影响效果, 结果表明, 德行领 导与总体创新行为、能力导向创新行为、行为导向创新行为均存在显著正相关, 分析结果与 现有文献数据保持一致, 也符合理论假设 $1 、 1 \mathrm{a} 、 1 \mathrm{~b}$ 。结果说明, 德行领导对构建高创新型企 业发挥重大的作用, 因此, 领导者在人际交往以及工作日常中应充分的展现高水平的道德素 质和人格魅力, 从而激发员工的追随、效仿、学习, 也是形成良好的企业创新氛围的重要因 素。跨文化情境、创新主体对德行领导与企业创新有效性之间的关系具有明显的调节效果。 其中, 德行领导对能力导向创新行为的影响高于对行为导向创新行为的影响。跨文化情境在 德行领导与多焦点创新行为的关系中起调节效应, 在中国情境下, 德行领导对企业创新、能 力导向企业创新的影响更强，可能是由于中国企业组织自古以来受到浓厚的中国儒家传统文 化和家庭观念的影响，企业领导普遍认可 “以身作则” “垂范百世” “德为人表” 的领导作 风，企业员工普遍认同 “投桃报李” “礼尚往来” “互利互惠” 的工作态度 ${ }^{[19]}$ ，然而，德行 领导具有公正无私、严于律己、赏罚分明、以身作则的特征, 当领导者在实践中表现出上述 的行为特征时, 员工会感受到公平公正, 更加的尊敬领导, 愿意为领导的行为和组织的发展 提供创造性或创新性的想法。同时领导的这些道德品质也将作为员工的学习榜样，他们将调 动自己的创造力, 通过榜样的力量来不断强化自身的业务水平 ${ }^{[20]}$ 。然而在西方的企业管理中 更加强调严格的等级制度, 标准化, 流程化的管理体系, 淡化对企业员工人性的关怀, 因而 员工在中国的文化情境下，会更加偏向于将创新行为看作是一种回报行为，用于回报领导的 德行领导。由于样本量的局限性, 未能检验德行领导与行为导向创新行为之间是否存在创新 主体为团队、组织的调节变量, 在一定程度上影响研究的全面性。另一方面, 由于西方学者 在德行领导研究领域的发展较慢, 本研究关于非中国情境下的样本量相对较少, 在某种程度 上会影响跨文化情境的调节作用, 其次, 德行领导作为家长式领导三个维度的重要组成部分, 未来的研究可以结合家长式领导的另外两个领导方式, 威权领导、仁慈领导进行与企业创新 有效性的深入研究。

\section{References}

[1] T. K. Amabile, E. A. Schatzela, and G. B. Monetaa, Leader behaviors and the work environment for creativity: Perceived leader support, Leadership Quarterly, vol. 15, pp. 5-32, 2004.

[2] D. Boucknooghe, C. Afa, and U. Raja, How ethical leadership shapes employees' job performance: the mediating roles of goal congruence and psychological capital, Journal of Business Ethics, vol. 129, pp. 251-264, 2015.

[3] M. E. Brown and M. S. Mitchell, Ethical and unethical leadership: exploring new avenues for future research, Business Ethics Quarterly, vol. 20, pp. 583-616, 2010.

[4] K. Byron and S. Khazanchi, Rewards and creative performance: a meta-analytic test of theoretically derived hypotheses, Psychological Bulletin, vol. 103, pp. 809-830, 2012.

[5] C. Boxun, Z. Lifan, and F. Jingli, Paternalistic leadership: the construction and measurement of ternary model, Research on Indigenous Psychology, vol. 14, pp. 3-36, 2000.

[6] P. M. Blau, Exchange and Power in Social Life, New York: Wiley, 1964.

[7] A. Gouldner, The norm of reciprocity, American Sociological Review, vol. 25, pp. 161-178, 1960.

[8] R. Cropanzano and M. S. Mitchell, Social exchange theory: an interdisciplinary review, Journal of Management, vol. 31, pp. 874-900, 2005. 
[9] R. Eisenberger, M. S. Rexwinkel, and B. Lynch, Reciprocation of perceived organizational support, Journal of Applied Psychology, vol. 86, pp. 42-51, 2001.

[10]P. W. Hom, A. S. Wu, and J. B. Lee, Explaining employment relationships with social exchange and job dilemma, Journal of Applied Psychology, vol. 94, pp. 277-297, 2009.

[11]W. Guolian and W. Haiying, The Influence of paternalistic leadership on organization's innovation performance, Research on Economy and Management, vol. 72, pp. 91-100, 2012

[12]Z. Fei, L. Cunpei, and S. Lui, Research on the relationship between moral leadership and organizational management innovation: the intermediary role of informal knowledge sharing, Management Review, vol. 27, pp. 169-177, 2015.

[13]X. Yangni, G. Xuanqin, and J. Wan, The impact of moral leadership on employees' creativity and performance: an empirical study based on LMX theory, Management Review, vol. 26, pp. 139-147, 2014.

[14] W. Hanyang, S. Jun, and H. Changqing, Moral leadership and employee creativity- the intermediary role of LMX and team identity, Soft Science, vol.31, pp. 76-80, 2017.

[15]W. Caoxue, Research on the Impact of Paternalistic Leadership on Employees' Behavior in Bootleg Innovation, China Labor, vol.58, pp. 58-69, 2019.

[16]W. Li and C. Yonhong, Paternalistic leadership and teachers' teaching innovation: the intermediary role of trust and self-motivation, Educational Research and Experiment, vol. 90, pp. 41-46, 2016.

[17] M. Yiyuan, C. Wenge, and W. Lihua, The effects of entrepreneurs' moral awareness and ethical behavior on product innovation of new ventures: evidence from China, Product Innovation, vol. 13, pp. 421-446, 2019.

[18] M. Koslowsky and A. Sagie, On the efficacy of credibility intervals as indicators of moderator effects in meta-analytic research, Journal of Organizational Behavior, vol. 14, pp. 695-699, 1993.

[19]F. Jingli and C. Boxun, Paternalistic leadership of Chinese organizations: an analysis of a cultural perspective, Research on Indigenous Psychology, vol. 14, pp. 127-180, 2000.

[20]P. Jansen, F. A. J. Van, and H. W. Volberda, Exploratory innovation, exploitative innovation, and performance: effects of organization antecedents and environment moderators, Management Science, vol. 52, pp. 1661-1674, 2006. 